\title{
Pharmacogenomics in the United States Community Pharmacy Setting: The Clopidogrel-CYP2C19 Example
}

\author{
David F Kisor' \\ Natasha J Petry (D) $^{2,3}$ \\ David R Bright $\mathbb{D}^{4}$
}

'Manchester University, Department of Pharmaceutical Sciences and Pharmacogenomics, Fort Wayne, IN, USA; ${ }^{2}$ North Dakota State University, College of Health Professions, Department of Pharmacy Practice, Fargo, ND, USA; ${ }^{3}$ Sanford Imagenetics, Sioux Falls, ND, USA; ${ }^{4}$ Ferris State University, Department of Pharmaceutical Sciences, Big Rapids, MI, USA
Correspondence: David F Kisor Manchester University, Department of Pharmaceutical Sciences and

Pharmacogenomics, 10627 Diebold Road, Fort Wayne, IN, 46845, USA

Tel + I 260-470-2747

Fax + I 260-470-4440

Email dfkisor@manchester.edu

\begin{abstract}
Pharmacogenomics (PGx) is expanding across health-care practice settings, including the community pharmacy. In the United States, models of implementation of PGx in the community pharmacy have described independent services and those layered on to medication therapy management. The drug-gene pair of clopidogrel-CYP2C19 has been a focus of implementation of PGx in community pharmacy and serves as an example of the evolution of the application of drug-gene interaction information to help optimize drug therapy. Expanded information related to this drug-gene pair has been provided by the US Food and Drug Administration and clinical PGx guidelines have and continue to be updated to support clinical decision-making. Most recently direct-to-consumer (DTC) PGx has resulted in patient generated sample collection and submission to a genetic testing-related company for analysis, with reporting of genotype and related phenotype information directly to the patient without a health-care professional guiding or even being involved in the process. The DTC testing approach needs to be considered in the development or modification of PGx service models in the community pharmacy setting. The example of clopidogrelCYP2C19 is discussed and current models of PGx implementation in the community pharmacy in the United States are presented. New approaches to PGx services are offered as implementation continues to evolve and may now include DTC information.
\end{abstract}

Keywords: pharmacogenomics, pharmacogenetics, clopidogrel, community pharmacy, implementation

\section{Introduction and Background of Pharmacogenomics Across Health-Care Settings}

Pharmacogenomics (PGx), as a part of precision medicine initiatives, is often implemented within health systems by multi-disciplinary teams; but pharmacists, in all settings, including the community pharmacy, are prime to lead such efforts. ${ }^{1-4}$ Cardiology, psychiatry, infectious disease, oncology and primary care are some examples of current practice areas for application of PGx as evidenced by available Clinical Pharmacogenetics Implementation Consortium (CPIC) guidelines ${ }^{5,6}$ but other areas of practice use PGx as well. ${ }^{7}$ While multiple barriers exist to implementing PGx, there are many examples of successful clinical implementation. ${ }^{2,8-15}$ The implementation of a full scale PGx program is a large undertaking and some programs start with implementation of a single gene, such as $C Y P 2 C 19$, for use as reactive testing in guiding clopidogrel dosing, ${ }^{2,16}$ or a single specialty area such as cardiology. ${ }^{17}$ Many programs have documented success implementing pre-emptive panel-based PGx programs. ${ }^{10,12,16,18-21}$ 
Laboratory considerations affect the use of PGx. Inhouse laboratories often offer benefits such as the ability for increased interoperability within electronic health records (EHR) for use of storage and automated clinical decision support, control over test content and results reporting, in addition to turn around time. ${ }^{8,22}$ External labs allow for health systems and community pharmacies to focus on clinical care while leaving the complexities of genetic laboratory maintenance to lab experts. While most clinical use of PGx is through provider-ordered testing, direct-to-consumer (DTC) PGx testing is also an option for patients to obtain some PGx information directly without requiring the involvement of a health-care professional. In the context of DTC, community pharmacists may be the first health care professional approached relative to PGx testing results.

PGx data is an important part of a patient's clinical picture, yet the literature continues to demonstrate that many pharmacists see the value of PGx but do not feel comfortable interpreting the data. ${ }^{23-26}$ Despite many pharmacists not feeling comfortable to utilize the data, the Oath of a Pharmacist includes "I will accept the lifelong obligation to improve my professional knowledge and competence"27 Multiple continuing education, certificate, degree, toolkit and training programs are available for pharmacists who seek further PGx educational learning opportunities. Pharmacists in multiple settings outside of precision medicine programs need an understanding of the varying levels of evidence for use of PGx data within clinical care and resources to help apply the data such as CPIC, the Pharmacogenomics Knowledgebase (PharmGKB), the Dutch Pharmacogenetics Working Group (DPWG) and United States Food and Drug Administration (FDA) tables of PGx information. ${ }^{28-31}$ As use of PGx expands, hospital, ambulatory and community pharmacists should be prepared to answer PGx-related medication questions. $^{32}$

Many successful inpatient/hospital implementations are documented in the literature..$^{2,10,11,14-16,19}$ Given the turnaround time of some PGx testing, especially in locations without on-site laboratory testing support, in-hospital PGx testing may not always be feasible. As such, data sharing and engagement with outpatient ambulatory care clinics and community pharmacy may be necessary. Barriers to active use of PGx in outpatient settings have been recognized, such as cost/reimbursement issues, time to devote to PGx-focused work, and pharmacist education. ${ }^{33}$ Despite these noted barriers, strategies for outpatient pharmacy- focused billing and reimbursement have been proposed and implemented; $;^{34-36}$ and time requirements in the clinical setting are modest. ${ }^{37,38}$

Outpatient ambulatory care settings often allow for the opportunity to interact with the patient in addition to having access to the EHR. Selected sites with established use of PGx in ambulatory care include NorthShore University HealthSystem's multidisciplinary PGx clinic within a community health system ${ }^{35}$ and Cleveland Clinic's two models of fee-for-service electronic consultation and ambulatory clinic. ${ }^{36}$ Sanford Health's Imagenetics program offers preemptive testing through primary care services and has PGx pharmacists embedded within Internal Medicine clinics. ${ }^{12}$ Mission Health developed an outpatient clinic for PGx consultations. ${ }^{39}$ University of Florida (UF) Health Precision Medicine Program implemented ambulatory care focused PGx across multiple UF Health and partner sites. ${ }^{1}$ Further growth is expected within ambulatory care with pilots such as MedStar Health's ambulatory-based pilot estimating PGx clinical actionability. $^{40}$

While the community pharmacy setting has long been a proposed site for integration of PGx into modern healthcare practices, similar barriers persist. ${ }^{41,42}$ However, recent studies have demonstrated the viability of PGx work in general in community pharmacies. ${ }^{43-45}$ Further, DTC PGx testing presents new opportunities for community pharmacists to be deeply involved in DTC PGx work. ${ }^{46}$ As DTC PGx testing becomes increasingly available, cost efficient, and visible in health systems and community pharmacies, clinical workflow models may need to further adapt to involve patient-gathered PGx data. In fact, as noted, community pharmacists may be the first clinicians in the care process to learn of PGx data if brought to the pharmacy by the patient via DTC testing. As such, community pharmacists may be responsible to ensure proper drug therapy changes are discussed and managed according to clinical guidelines. Information about community pharmacy patient perceptions has been obtained that may help to drive communication strategies with patients in this setting to help ensure therapeutic optimization commensurate with PGx data available. ${ }^{47,48}$

A number of community pharmacy-based PGx models have been presented and discussed in the literature as PGx-focused practice has evolved over the last decade. ${ }^{45,49-51}$ Similar to many implementation strategies in other clinical settings, these community pharmacy models have used a single drug-gene pair as an initial 
implementation strategy, notably with a focus on managing the clopidogrel-CYP2C19 interaction. ${ }^{49-51}$ In this paper, we describe "pharmacogenomics in the community pharmacy", relative to experience in the United States, by examining the pharmacogenomics history of clopidogrel and pharmacy services related to optimizing antiplatelet therapy, with specific discussion on the more recent implications of DTC PGx testing on clinical opportunities and clinical workflow considerations.

\section{History of Clopidogrel PGx}

Clopidogrel is an example of a drug which has a clear PGx relationship. ${ }^{52-54}$ The prodrug is metabolized in sequential steps by the enzyme product (CYP2C19) of the polymorphic CYP2C19 gene. $^{55}$ The risk of major adverse cardiovascular events (MACE) in the setting of antiplatelet therapy, relative to percutaneous coronary intervention (PCI), is recognized across health-care settings, ie, from the "cath lab" to the community pharmacy. Decreasing the potential risk of MACE can be accomplished by knowing a patient's PGx prior to catheterization and if not, by applying PGx as soon as possible post PCI, such as through community pharmacy services, to optimize antiplatelet therapy. Technology exists to allow for rapid turn-around of CYP2C19 genotypes in the catheterization lab, although in many settings the throughput of the instrumentation may be limiting, eg, when multiple patients need testing simultaneously, or there is a sample issue. In these cases, utilizing community pharmacy services can be beneficial. This technology may be applicable in the community pharmacy setting, but the approach needs to be fully examined.

\section{US Food and Drug Administration Approved Labeling}

Clopidogrel (Plavix ${ }^{\circledR}$ ) was originally approved for marketing in the United States on November 17, 1997 for "reduction of atherosclerotic events". ${ }^{56}$ In the original package labeling, the metabolism of clopidogrel was described as

In vitro and in vivo, clopidogrel undergoes rapid hydrolysis into its carboxylic acid derivative. In plasma and urine, the glucuronide of the carboxylic acid is also observed. ${ }^{56}$

In vitro information related to high concentrations of clopidogrel inhibiting the metabolism of CYP2C9 substrate drugs was noted. ${ }^{56}$ However, at this time, the specific metabolic pathways were not discussed and certainly there was no mention of the influence of CYP2C19 variants on metabolism, ie, sequential conversion to the active form. In 2009, the US FDA-approved clopidogrel package labeling was updated to include information related to CYP2C19 phenotypes in the pharmacogenetics subsection of the clinical pharmacology section of the label. ${ }^{57}$ This label noted the percentage of poor metabolizers (PMs) in White, Black, and Chinese populations to be $2 \%, 4 \%$, and $14 \%$, respectively. ${ }^{57}$ The prevalence of PMs was significant and a "boxed warning" was added in March of 2010, which described "reduced effectiveness in patients who are poor metabolizers of Plavix". ${ }^{58,59}$ The boxed warning also referred to poor metabolizers not effectively converting Plavix to its active form. Additionally, health-care professionals were informed of testing availability to identify genetic-based differences in CYP2C19 function. Finally, in 2016, the approved label informed health-care professionals to consider alternative antiplatelet agents in CYP2C19 PMs. ${ }^{59}$ Although the wording has changed, the current FDA-approved package labeling boxed warning conveys the same information as the original 2010 boxed warning. ${ }^{56}$

\section{Clinical Guidelines/Recent Trial}

While the updated 2010 FDA-approved package labeling of Plavix noted decreased antiplatelet effectiveness in CYP2C19 PMs, literature reviews by independent pharmacogenetics expert groups resulted in the publication of clinical guidelines which expanded our knowledge related to metabolism phenotypes and response to clopidogrel. ${ }^{60,61}$ Both CPIC and the DPWG provided recommendations to either avoid or consider avoiding the use of clopidogrel in intermediate metabolizers (IMs) in addition to PMs. ${ }^{60,61}$ Discussion around the data related to the impact of the $* 2$ variant (no function) is somewhat controversial, especially in the IM group with low risk of adverse cardiovascular events. However, a cogent argument was made describing the current literature at the time and concluded that specific higher-risk PCI patients who are $* 2$ carriers would benefit from genotype guided antiplatelet therapy. ${ }^{62}$ Most recently, the TAILOR-PCI Randomized Clinical Trial found no statistically significant difference in a composite end point of major cardiovascular or neurological events (ie, cardiovascular death, myocardial infarction, stroke, stent thrombosis, and severe recurrent ischemia) when comparing CYP2C19 genotypeguided antiplatelet therapy to conventional therapy, 
relative to patients carrying at least one no function allele (ie, *2, *3) ${ }^{54}$ However, the primary endpoint of the trial was $50 \%$ reduction at one year in the combined rate of major adverse effects, a goal that goes beyond what would be acceptable clinically. In fact, it was noted that during the higher risk first three months, there was an $80 \%$ reduction in the rate of adverse events in the genotypeguided group. ${ }^{63,64}$ Overall, there was a $34 \%$ reduction in major adverse events at one year and although not specifically powered for this percentage, these findings support the use of genotype-guided antiplatelet therapy. ${ }^{63,64}$ The Tailor PCI trial compares favorably with the "POPular Genetics Trial", which had two primary outcomes measures including combined outcome of net adverse clinical events and bleeding (CABG-related and non-CABGrelated or minor bleeding at 12 months). ${ }^{65}$ The intentionto-treat study design and noninferiority analysis showed that genotype-guided antiplatelet therapy was noninferior to standard therapy, with actually fewer cases of adverse clinical events as well as a lower incidence of bleeding events in the genotype-guided group. ${ }^{65}$ Additionally, a meta-analysis identified 1335 studies of which 12 were included in analysis. Seven of these were randomized controlled trials. ${ }^{66}$ There were nearly 16,000 patients across the included studies. ${ }^{66}$ The analysis found that genotyping for CYP2C19 was warranted when considering the use of oral P2Y12 inhibitor therapy to differentiate the use of clopidogrel and other agents. ${ }^{66}$ The CPIC clopidogrel-CYP2C19 guideline was last updated in 2013 and the DPWG guidelines prior to that year. Currently, CPIC is updating the clopidogrel-CYP2C19 guideline again to include information from the Tailor-PCI trial as well as other up-to-date information beyond the 2013 publication.

\section{Direct to Consumer PGx}

The evolution of CYP2C19 genotyping relative to antiplatelet therapy has a very significant direct-to-consumer (DTC) context. For many years, the DTC company 23andMe had been providing genotyping results including results related to PGx. The 23andMe website provided information regarding CYP2C19 and clopidogrel and other pharmacogenomic information. Additionally, users could download their raw data and gain access to information of literally hundreds of thousands of variants, including further PGx data. In November of 2013, the US FDA ordered 23andMe to shut down sales of its testing kits and resulted in discontinuation of providing consumers with information related to their genetics, including PGx information. ${ }^{67}$ Following continued interaction with the FDA related to PGx, 23andMe gained marketing approval in October of 2018 for its "Personal Genome Service (PGS) Pharmacogenetic Reports", but language specifically noted that health-care providers "should not use the test to make any treatment decisions". ${ }^{68}$ Additionally, the FDA stated "Results from this test should be confirmed with independent pharmacogenetic testing before making any medical decisions." ${ }^{\circ 8}$ At the time this approval was to spur on discussion about PGx between the patient and their health-care professionals. This cracked open the door for patients to get their genetic information related to PGx directly, without a health-care intermediary. In August of 2020, the door to direct clinical use of 23andMe-provided data was pushed wide open as the FDA approved the use of the 23andMe DTC PGS report, without independent lab confirmation, for clinical decision support relative to clopidogrel and citalopram. ${ }^{69}$ With this ruling, patients can now bring their reports to any healthcare professional, including community pharmacists, who may use the CYP2C19 information to inform antiplatelet therapy.

\section{Clopidogrel PGx and Community Pharmacy Models}

\section{Published Models}

While hospital-based care models involving PGx gained popularity shortly after the FDA changed the labeling for clopidogrel to include notation about PGx, and initial successes were demonstrated in larger medical centers, viability of widespread replication of the hospital-based models came into question when on-site, rapid turn-around PGx testing was not immediately available. For instance, if the PGx testing started in the hospital, but results did not come back until after the patient was discharged, how would follow-up take place? Who needs to be "in the loop" with PGx-guided therapy, particularly if prescription insurance companies set up copay tiers that favor use of clopidogrel? If PGx testing does not take place in the hospital, which health-care professionals could reasonably conduct PGx testing and facilitate information sharing and clinical decision-making by the prescriber?

These questions, among others, led to the advocacy for and development of community pharmacy-based models for PGx-focused management of clopidogrel following release of the associated CPIC and other guidelines. ${ }^{41-45,49-51,70-72}$ Multiple models of care were 
initially described, not as a substitute for hospital-based models, but as a way to augment patient transition of care when local hospitals did not offer rapid turn-around PGx testing services. In the first two community pharmacyfocused models reported in the literature, it was generally assumed that the pharmacist would be the person in the health-care system that becomes responsible for obtaining PGx data. As such, the first approach takes place in such a way that the pharmacist may hold a medication therapy management (MTM) appointment with a patient where concepts surrounding PGx are discussed and a test is administered to the patient. The pharmacist would then bring the patient back in for a second MTM encounter following the receipt of PGx data and complete any clinical recommendation work that was required so that the information could be delivered to the patient. ${ }^{49,50,70}$ In the second approach, the first MTM encounter is held in a consistent fashion, but the second MTM encounter is replaced by a phone call that would be used to change therapy if necessary. Given that antiplatelet therapy may not change for many patients based on PGx data, there may be some efficiencies to only holding a second MTM encounter if there is a more pressing need for further intervention or education. ${ }^{51,71}$

A third model was later suggested that involved pharmacists directly performing PGx testing in the community pharmacy in the presence of the requisite PGx testing equipment that offered results in approximately an hour. In particular, if the pharmacist was equipped with authority (independently or via protocol/collaborative practice agreement with the prescribing physician) to independently adjust therapy based on PGx results, the time required for this process may not be substantially more than the previously described workflow models. ${ }^{37}$

\section{Community Pharmacy Clinical Models: Moving Forward}

Recent advances in DTC PGx testing have obviously created new models for PGx data to be obtained and provided to the health-care system. Given that DTC PGx tests are often sold in community pharmacies, and as community pharmacists are some of the most accessible health-care professionals, DTC PGx testing has opened the door to additional potential for community pharmacist engagement. Whereas pharmacists previously had to be the recipient of a PGx test result from another medical provider or had to facilitate the ordering of a PGx test within the pharmacy in order to obtain PGx data, the potential sources for receiving PGx data are broadening. Figure 1 describes the modern pathways for data input into clinical workflow relative to antiplatelet therapy as an example.

Following the rapid increase in DTC PGx and the increased potential that the patient may be responsible for bringing PGx data into the path of care, several potential scenarios could be observed where a patient may bring the PGx information to the pharmacist as the initial access point to the health-care system. For instance, a patient may obtain their own PGx data through a DTC test and go directly to the internet for interpretation of next steps. The patient may then bring whatever information was gathered online to the community pharmacist with a request for intervention. Given that the complexity of PGx may often require clinical information beyond simply genotype/phenotype data in order to make a proper clinical decision (eg, active/concurrent disease states, concurrent medication use), the patient may not always arrive at the correct therapeutic conclusion by internet-based research alone, and the pharmacist may be well suited to help with patient counseling and education. ${ }^{62,72}$ The pharmacist, acting as a member of the patient's health-care team can provide the patient-received information to the prescribing physician for further consideration of patient care. Similarly, the patient may simply bring the PGx report to the pharmacy and ask the pharmacist for assistance with PGx data interpretation in the context of their current medications. This may be beneficial for the patient and the physician-led health-care team, as the team may become aware of PGx data that were not previously known, and the patient may come to have an optimized pharmacotherapeutic regimen based on PGx information. However, the community pharmacist may have to take on a lot of communication work with the health-care system, as few published instances exist where PGx information comes into the health-care system from the patient directly, and the novelty of this approach may be met with unfamiliarity by clinicians. Further, there are no clear standard approaches for community pharmacists to introduce clinical laboratory data into EHR systems, so data sharing logistics must also be considered when the pharmacist is the initial recipient of DTC PGx data.

In addition, while the models above describe that the community pharmacist can obtain the PGx data and make clinical decisions from there, community pharmacists and other health-care professionals must be aware that PGx 


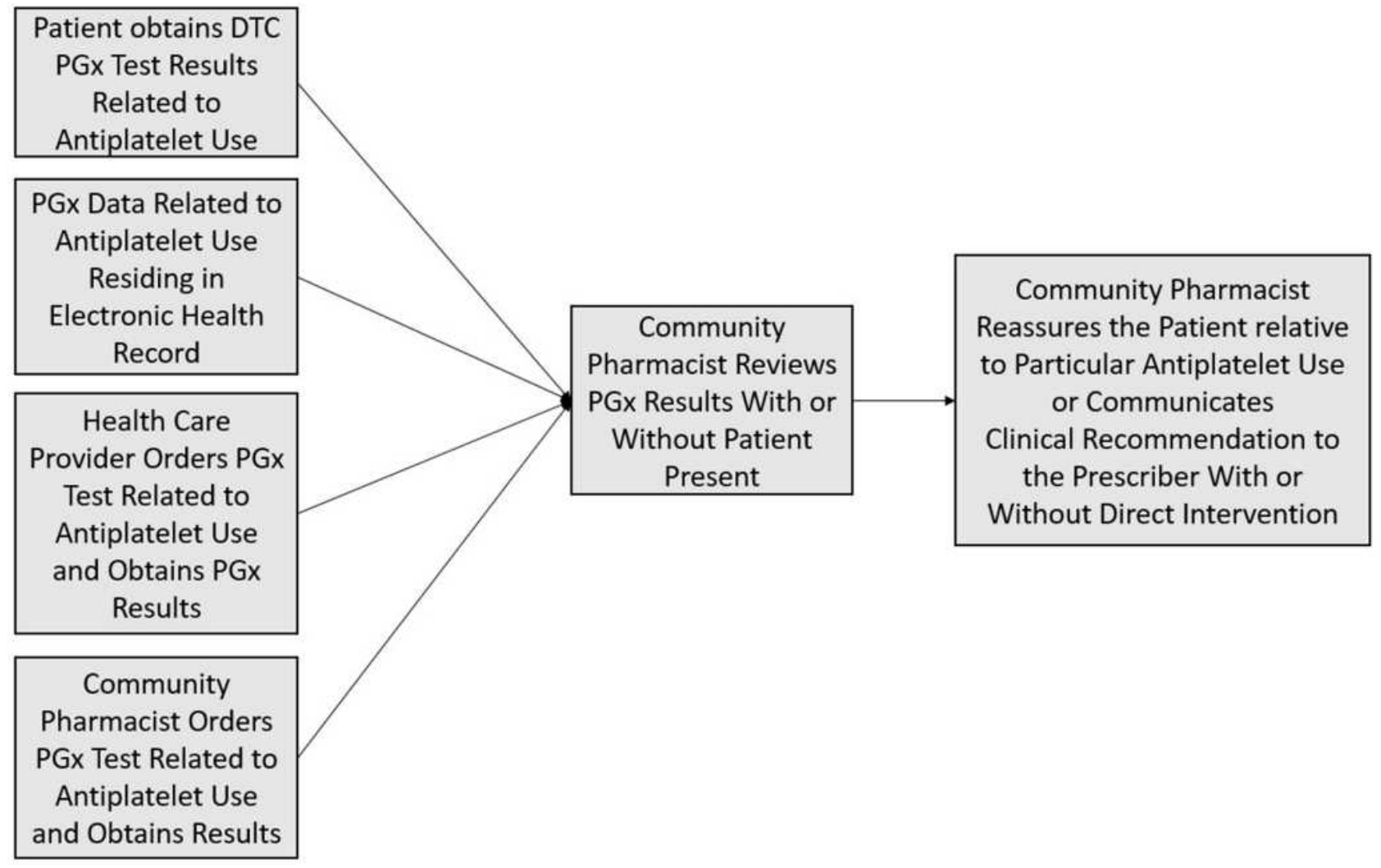

Figure I Clinical workflow models for community pharmacist engagement.

data does not change over the lifespan of the patient. With roughly a decade of clinical PGx experience in the healthcare system, pharmacists must understand that a patient's genotype and phenotype information for key pharmacogenes may already be in existence in the health-care system. ${ }^{46}$ Efforts need to be made to share and flag such data throughout the health-care system so clinicians do not make clinical recommendations that would go against what would be appropriate had PGx information been known.

Regardless of whether the PGx data originate from the patient via DTC testing, from the pharmacy via in-pharmacy testing, or via another health-care entity/lab, PGx data sharing is critical to ensure that the data is used consistently; and unnecessary and costly repeat testing is avoided. While models for data sharing have been proposed, even the simplest approach of sharing, a PDF report, may bring challenges. ${ }^{73}$ For instance, if the report is provided directly to the patient, the red (do not use a given drug)/yellow (use a given drug with caution)/ green (use a given drug) context may be misinterpreted as a need for immediate discontinuation of a medication. Not all red, yellow, or green indications in a report necessitate an immediate medication discontinuation or change. Pharmacists in the community setting must be adequately prepared to help patients interpret PGx data that they may have, either through a formal test ordered by a health-care professional, or by a DTC test provided to the patient.

\section{Summary}

Models of PGx services have been evaluated across health-care settings, with pharmacy being integral to their implementation. Pharmacists will continue to play a critical role in the appropriate application of PGx as testing expands beyond the traditional health-care locations. Community pharmacy models, first tested in the context of clopidogrel/CYP2C19, will expand to include other drug-gene pairs. Pharmacogenomics in concert with MTM will allow for further refinement and optimization of pharmacotherapy. Direct to consumer PGx data will drive further interaction between patients and pharmacists and will necessitate increased communication between healthcare providers. New models of PGx implementation will be necessary to address different approaches to testing and 
the community pharmacy will be a vital setting for application of PGx.

\section{Disclosure}

DRB has conducted funded research with Genemarkers, LLC and reports a pending patent. The authors report no other conflicts of interest in this work.

\section{References}

1. Cicali EJ, Weitzel KW, Elsey AR, et al. Challenges and lessons learned from clinical pharmacogenetic implementation of multiple gene-drug pairs across ambulatory care settings. Genet Med. 2019;21(10):2264-2274. doi:10.1038/s41436-019-0500-7.

2. Weitzel KW, Elsey AR, Langaee TY, et al. Clinical pharmacogenetics implementation: approaches, successes, and challenges. $\mathrm{Am} \mathrm{J} \mathrm{Med}$ Genet C Semin Med Genet. 2014;166C(1):56-67. doi:10.1002/ajmg. c. 31390 .

3. American Society of Health System Pharmacists. ASHP statement on the pharmacist's role in clinical pharmacogenomics. Am J Health Syst Pharm. 2015;72(7):579-581. doi:10.2146/sp150003.

4. Zellmer WA, ed. Pharmacy Forecast 2016-2020: Strategic Planning Advice for Pharmacy Departments in Hospitals and Health Systems, December 2015. Bethesda, MD: ASHP Research and Education Foundation; 2017. Available from: https://www.ashpfoundation.org/-/ media/REF/Research/PDFs/2016 PharmacyForecast Report.ashx?la= en\&hash=50295F3AF81810FC184EB67E2793CD3ED6AC8754. Accessed February 28, 2021.

5. The clinical pharmacogenetics implementation consortium $\left(\mathrm{CPIC}^{\circledR}\right)$. Guidelines. Available from: https://cpicpgx.org/guidelines/. Accessed February 28, 2021.

6. Relling MV, Klein TE, Clinical Pharmacogenetics CPIC. Implementation consortium of the pharmacogenomics research network. Clin Pharmacol Ther. 2011;89(3):464-467. doi:10.1038/ clpt.2010.279.

7. Caudle KE, Gammal RS, Karnes JH, et al. PRN OPINION PAPER: application of precision medicine across pharmacy specialty areas. J Am Coll Clin Pharm. 2019;2(3):288-302. doi:10.10 $02 /$ jac5.1107

8. Liu M, Vnencak-Jones CL, Roland BP, et al. A tutorial for pharmacogenomics implementation through end-to-end clinical decision support based on ten years of experience from PREDICT. Clin Pharmacol Ther. 2021;109(1):101-115. doi:10. $1002 /$ cpt.2079.

9. Sissung TM, McKeeby JW, Patel J, et al. Pharmacogenomics implementation at the national institutes of health clinical center. $J$ Clin Pharmacol. 2017;57:S67-S77. doi:10.1002/jcph.993

10. Dunnenberger HM, Crews KR, Hoffman JM, et al. Preemptive clinical pharmacogenetics implementation: current programs in five US medical centers. Annu Rev Pharmacol Toxicol. 2015;55(1):89-106. doi:10.1146/annurev-pharmtox-010814-124835.

11. Wang L, Weinshilboum R. Pharmacogenomics in practice. Clin Pharmacol Ther. 2019;106(5):936-938. doi:10.1002/cpt.1600.

12. Petry N, Baye J, Aifaoui A, et al. Implementation of wide-scale pharmacogenetic testing in primary care. Pharmacogenomics. 2019;20(12):903-913. doi:10.2217/pgs-2019-0043.

13. Manzi SF, Fusaro VA, Chadwick L, et al. Creating a scalable clinical pharmacogenomics service with automated interpretation and medical record result integration - experience from a pediatric tertiary care facility. J Am Med Inform Assoc. 2017;24(1):74-80. doi:10.1093/ jamia/ocw052.
14. Cavallari LH, Weitzel KW, Elsey AR, et al. Institutional profile: University of Florida health personalized medicine program. Pharmacogenomics. 2017;18(5):421-426. Epub 2017 Mar 27. doi:10.2217/pgs-2017-0028.

15. Ramsey LB, Prows CA, Zhang K, et al. Implementation of pharmacogenetics at Cincinnati Children's Hospital Medical Center: lessons learned over 14 years of personalizing medicine. Clin Pharmacol Ther. 2019;105(1):49-52. doi:10.1002/cpt.1165.

16. Johnson JA, Elsey AR, Clare-Salzler MJ, Nessl D, Conlon M, Nelson DR. Institutional profile: University of Florida and Shands Hospital personalized medicine program: clinical implementation of pharmacogenetics. Pharmacogenomics. 2013;14(7):723-726. doi:10.2217/pgs.13.59.

17. Empey PE, Stevenson JM, Tuteja S, et al.; IGNITE Network. Multisite investigation of strategies for the implementation of CYP2C19 genotype-guided antiplatelet therapy. Clin Pharmacol Ther. 2018;104(4):664-674. doi:10.1002/cpt.1006.

18. Van Driest SL, Shi Y, Bowton EA, et al. Clinically actionable genotypes among 10,000 patients with preemptive pharmacogenomic testing. Clin Pharmacol Ther. 2014;95(4):423-431. doi:10.1038/ clpt.2013.229.

19. Hoffman JM, Haidar CE, Wilkinson MR, et al. PG4KDS: a model for the clinical implementation of pre-emptive pharmacogenetics. Am $J$ Med Genet. 2014;166C(1):45-55. doi:10.1002/ajmg.c.31391

20. Ji Y, Skierka JM, Blommel JH, et al. Preemptive pharmacogenomic testing for precision medicine: a comprehensive analysis of five actionable pharmacogenomic genes using next-generation DNA sequencing and a customized CYP2D6 genotyping cascade. J Mol Diagn. 2016;18(3):438-445. doi:10.1016/j. jmoldx.2016.01.003.

21. Haidar CE, Relling MV, Hoffman JM. Preemptively precise: returning and updating pharmacogenetic test results to realize the benefits of preemptive testing. Clin Pharmacol Ther. 2019;106(5):942-944. doi:10.1002/cpt.1613.

22. Vo TT, Bell GC, Owusu Obeng A, Hicks JK, Dunnenberger HM. Pharmacogenomics implementation: considerations for selecting a reference laboratory. Pharmacotherapy. 2017;37(9):1014-1022. doi:10.1002/phar.1985.

23. Nagy M, Eirini Tsermpini E, Siamoglou S, Patrinos GP. Evaluating the current level of pharmacists' pharmacogenomics knowledge and its impact on pharmacogenomics implementation. Pharmacogenomics. 2020;21(16):1179-1189. doi:10.2217/pgs-2020-0076.

24. Tuteja S, Haynes K, Zayac C, Sprague JE, Bernhardt B, Pyeritz R. Community pharmacists' attitudes towards clinical utility and ethical implications of pharmacogenetic testing. Pers Med. 2013;10(8):793800. 7. doi: $10.2217 / \mathrm{pme} .13 .85$

25. Schwartz EJ, Issa AM. The role of hospital pharmacists in the adoption and use of pharmacogenomics and precision medicine. Pers Med. 2017;14(1):27-35. doi:10.2217/pme-2016-0063

26. Hundertmark ME, Waring SC, Stenehjem DD, et al. Pharmacist's attitudes and knowledge of pharmacogenomics and the factors that may predict future engagement. Pharm Pract (Granada). 2020;18 (3):2008. doi:10.18549/PharmPract.2020.3.2008.

27. American Pharmacists Association. Oath of a pharmacist. Available from: https://www.pharmacist.com/About/Oath-of-a-Pharmacist. Accessed May 6, 2021.

28. Caudle KE, Gammal RS, Whirl-Carrillo M, Hoffman JM, Relling MV, Klein TE. Evidence and resources to implement pharmacogenetic knowledge for precision medicine. Am J Health Syst Pharm. 2016;73(23):1977-1985. doi:10.2146/ajhp150977.

29. McDonagh EM, Whirl-Carrillo M, Garten Y, Altman RB, Klein TE. From pharmacogenomic knowledge acquisition to clinical applications: the PharmGKB as a clinical pharmacogenomic biomarker resource. Biomark Med. 2011;5(6):795-806. doi:10.2217/bmm.11.94. 
30. US FDA. Table of pharmacogenomic biomarkers in drug labeling. Available from: https://www.fda.gov/drugs/science-and-researchdrugs/table-pharmacogenomic-biomarkers-drug-labeling. Accessed February 28, 2021.

31. US FDA. Table of pharmacogenetic associations. Available from: https://www.fda.gov/medical-devices/precision-medicine/table-phar macogenetic-associations. Accessed February 28, 2021.

32. Roederer MW, Kuo GM, Kisor DF, et al. Pharmacogenomics competencies in pharmacy practice: a blueprint for change. $J$ Am Pharm Assoc. 2017;57(1):120-125. doi:10.1016/j.japh.2016.08.014.

33. Alexander KM, Divine HS, Hanna CR, Gokun Y, Freeman PR. Implementation of personalized medicine services in community pharmacies: perceptions of independent community pharmacists. J Am Pharm Assoc. 2014;54(5):510-517. doi:10.1331/JAPhA.20 14.13041

34. O'Connor SK, Michaels N, Ferreri S. Expansion of pharmacogenomics into the community pharmacy: billing considerations. Pharmacogenomics. 2015;16(3):175-180. doi:10.2217/pgs.14.183

35. Dunnenberger HM, Biszewski M, Bell GC, et al. Implementation of a multidisciplinary pharmacogenomics clinic in a community health system. Am J Health Syst Pharm. 2016;73(23):1956-1966. doi:10. 2146/ajhp160072.

36. Hicks JK, Stowe D, Willner MA, et al. Implementation of clinical pharmacogenomics within a large health system: from electronic health record decision support to consultation services. Pharmacotherapy. 2016;36(8):940-948. doi:10.1002/phar.1786.

37. Bright DR, Klepser M, Murry L, Klepser D. Pharmacist provided pharmacogenetic point of care testing consultation service: a time and motion study. J Pharm Technol. 2018;34(4):139-143. doi:10.1177/ 8755122518756651

38. Moaddeb J, Mills R, Haga SB. Community pharmacists' experience with pharmacogenetic testing. J Am Pharm Assoc. 2015;55 (6):587-594. doi:10.1331/JAPhA.2015.15017

39. Dressler LG, Bell GC, Ruch KD, Retamal JD, Krug PB, Paulus RA. Implementing a personalized medicine program in a community health system. Pharmacogenomics. 2018;19(17):1345-1356. doi:10. 2217/pgs-2018-0130.

40. Smith DM, Peshkin BN, Springfield TB, et al. Pharmacogenetics in practice: estimating the clinical actionability of pharmacogenetic testing in perioperative and ambulatory settings. Clin Transl Sci. 2020;13(3):618-627. doi:10.1111/cts.12748.

41. Padgett L, O’Connor S, Roederer M, McLeod H, Ferreri S. Pharmacogenomics in a community pharmacy: ACT now. $J \mathrm{Am}$ Pharm Assoc. 2011;51(2):189-193. doi:10.1331/JAPhA.2011.10178

42. Owen JA. Integrating pharmacogenomics into pharmacy practice via medication therapy management. J Am Pharm Assoc. 2011;51(6): e64-e74. doi:10.1331/JAPhA.2011.11543

43. Haga SB, Moaddeb J, Mills R, Voora D. Assessing feasibility of delivering pharmacogenetic testing in a community pharmacy setting. Pharmacogenomics. 2017;18(4):327-335. doi:10.2217/pgs-20160175

44. Papastergiou J, Tolios P, Li W, Li J. The innovative Canadian pharmacogenomic screening initiative in community pharmacy (ICANPIC) Study. J Am Pharm Assoc. 2017;57(5):624-629. doi:10.1016/j.japh.2017.05.006

45. Breaux S, Desrosiers FAD, Niera $M$, Sinha S, Nislow C. Pharmacogenomics at the point of care: a community pharmacy project in British Columbia. J Pers Med. 2021;11(1):11. doi:10.3390/jpm11010011

46. Gammal RS, Mayes J, Caudle KE. Ready or not, here it comes: direct-to-consumer pharmacogenomic testing and its implications for community pharmacists. $J$ Am Pharm Assoc. 2019;59 (5):646-650. doi:10.1016/j.japh.2019.06.008

47. Bright DR, Worley M, Porter B. Patient perceptions of pharmacogenomic testing in the community pharmacy setting. Res Soc Admin Pharm. 2021;17(4):744-749. doi:10.1016/j.sapharm.2020.06.022
48. Gibson ML, Hohmeier KC, Smith CT. Pharmacogenomics testing in a community pharmacy: patient perceptions and willingness-to-pay. Pharmacogenomics. 2017;18(3):227-233. doi:10.2217/pgs-2016-0161

49. O’Connor SK, Ferreri SP, Michaels NM, et al. Exploratory planning and implementation of a pilot pharmacogenetic program in a community pharmacy. Pharmacogenomics. 2012;13(8):955-962. doi:10.2217/pgs.12.67.

50. Ferreri SP, Greco AJ, Michaels NM, et al. Implementation of a pharmacogenomics service in a community pharmacy. $J \mathrm{Am}$ Pharm Assoc. 2014;54(2):172-180. doi:10.1331/JAPhA.2014.13 033.

51. Bright DR, Kisor DF, Smith A, Conaway M, Yu M. Implementation of a pharmacogenetic management service for post-myocardial infarction care in a community pharmacy. Pers Med. 2015;12 (4):319-325. doi:10.2217/pme.15.7

52. Brown SA, Pereira N. Pharmacogenomic impact of CYP2C19 variation on clopidogrel therapy in precision cardiovascular medicine. J Pers Med. 2018;8(1):8. doi:10.3390/jpm8010008.

53. Roden DM. Clopidogrel pharmacogenetics - why the wait? $N$ Engl $J$ Med. 2019;381(17):1677-1678. doi:10.1056/NEJMe1911496.

54. Pereira NL, Farkouh ME, So D, et al. Effect of genotype-guided oral P2Y12 inhibitor selection vs conventional clopidogrel therapy on ischemic outcomes after percutaneous coronary intervention: the TAILOR-PCI randomized clinical trial. JAMA. 2020;324 (8):761-771. doi:10.1001/jama.2020.12443.

55. Clopidogrel. DailyMed. Available from: https://dailymed.nlm.nih. gov/dailymed/drugInfo.cfm?setid=01b14603-8f29-4fa3-8d7e9d523f802e0b. Accessed February 3, 2021.

56. Approval Package for Plavix. U.S. Food and Drug Administration. Center for drug evaluation and research; November 17, 1997. Available from: https://www.accessdata.fda.gov/drugsatfda_docs/ nda/pre96/020839_s000.pdf. Accessed February 4, 2021.

57. Plavix ${ }^{\circledR}$ (clopidogrel bisulfate tablets). U.S. Food and Drug Administration. Available from: https://www.accessdata.fda.gov/drug satfda_docs/label/2009/020839s0401bl.pdf. Accessed February 5, 2021.

58. FDA Drug Safety Communication. Reduced effectiveness of plavix (clopidogrel) in patients who are poor metabolizers of the drug. Available from: https://www.fda.gov/drugs/postmarket-drug-safetyinformation-patients-and-providers/fda-drug-safety-communicationreduced-effectiveness-plavix-clopidogrel-patients-who-are-poor. Accessed February 5, 2021.

59. Plavix ${ }^{\circledR}$ (clopidogrel bisulfate tablets). U.S. Food and Drug Administration. Available from: https://www.accessdata.fda.gov/drug satfda_docs/label/2010/020839s042lbl.pdf. Accessed February 5, 2021.

60. Swen JJ, Nijenhuis M, de Boer A, et al. Pharmacogenetics: from bench to byte-an update of guidelines. Clin Pharmacol Ther. 2011;89 (5):662-673. doi:10.1038/clpt.2011.34.

61. Scott SA, Sangkuhl K, Gardner EE, et al. Clinical pharmacogenetics implementation consortium guidelines for cytochrome P450-2C19 (CYP2C19) genotype and clopidogrel therapy. Clin Pharmacol Ther. 2011;90(2):328-332. doi:10.1038/clpt.2011.132.

62. Johnson JA, Roden DM, Lesko LJ, Ashley E, Klein TE, Shuldiner AR. Clopidogrel: a case for indication-specific pharmacogenetics. Clin Pharmacol Ther. 2012;91(5):774-776. doi:10.1038/clpt.2012.21.

63. American College of Cardiology. TAILOR-PCI: genotype-guided antiplatelet therapy post PCI misses mark. Available from: https:// www.acc.org/latest-in-cardiology/articles/2020/03/24/16/41/sat-9amtailor-pci-clinical-implementation-clopidogrel-pharmacogenetics-acc2020. Accessed March 25, 2021.

64. Hughes S. TAILOR-PCI: clopidogrel genotyping trial narrowly misses endpoint. Available from: https://www.medscape.com/viewar ticle/927712. Accessed February 9, 2021. 
65. Claassens DMF, Vos GJA, Bergmeijer TO, et al. A genotype-guided strategy for oral P2Y12 inhibitors in primary PCI. $N$ Engl J Med. 2019;381(17):1621-1631. doi:10.1056/NEJMoa1907096.

66. Pereira NL, Rihal C, Lennon R, et al. Effect of CYP2C19 genotype on ischemic outcomes during oral P2Y12 inhibitor therapy: a meta-analysis. J Am Coll Cardiol. 2021;14(7):739-750. doi:10.10 16/j.jcin.2021.01.024

67. Brandom R. Body blow: how 23andMe brought down the FDA's wrath. Available from: https://www.theverge.com/2013/11/25/ 5144928/how-23andme-brought-down-fda-wrath-personal-geneticswojcicki. Accessed February 9, 2021.

68. FDA News Release. FDA authorizes first direct-to-consumer test for detecting genetic variants that may be associated with medication metabolism. Available from: https:/www.fda.gov/news-events/pressannouncements/fda-authorizes-first-direct-consumer-test-detectinggenetic-variants-may-be-associated-medication\#: :text=Today $\% 2 \mathrm{C} \%$ 20the \%20U.S.\%20Food\%20and,to\%20metabolize\%20some $\% 20$ medi cations\%20to. Accessed May 3, 2021.
69. U.S. Food and Drug Administration. Letter to 23andMe Inc. Available from: https://www.accessdata.fda.gov/cdrh_docs/pdf19/ K193492.pdf. Accessed February 9, 2021.

70. O'Connor SK, Ferreri SP, Michaels NM, et al. Making pharmacogenetic testing a reality in a community pharmacy. $J$ Am Pharm Assoc. 2012;52(6):e259-65. doi:10.1331/JAPhA.2012.12108

71. Kisor DF, Bright DR, Conaway M, Bouts BA, Gerschutz GP. Pharmacogenetics in the community pharmacy: thienopyridine selection post-coronary artery stent placement. J Pharm Pract. 2014;27 (4):416-419. doi:10.1177/0897190014522496

72. Bright DR, Greco AJ, Langervelt A, Kisor DF. Clinical implementation of personalized medicine in community pharmacies: considerations for clinicians. Per Med. 2017;14(6):471-475. doi:10.2217/pme-2017-0050

73. Hilden MP, Bright DR, Kisor DF, Christensen H. Pharmacogenomics: a practical primer for senior care pharmacists. Sr Care Pharm. 2019;34(6):363-369. doi:10.4140/TCP.n.2019.363

\section{Publish your work in this journal}

Pharmacogenomics and Personalized Medicine is an international, peer-reviewed, open access journal characterizing the influence of genotype on pharmacology leading to the development of personalized treatment programs and individualized drug selection for improved safety, efficacy and sustainability. This journal is indexed on the American Chemical Society's Chemical Abstracts Service (CAS). The manuscript management system is completely online and includes a very quick and fair peer-review system, which is all easy to use. Visit http://www.dovepress.com/testimonials.php to read real quotes from published authors. 